\title{
ESTUDO DA REAÇÃO DE OXIDAÇÃO PREFERENCIAL DO CO SOBRE O SISTEMA CuO/CeO ${ }_{2}-\mathrm{TiO}_{2}$
}

\author{
Cristhiane Guimarães Maciel* e José Mansur Assaf \\ Departamento de Engenharia Química, Universidade Federal de São Carlos, Rod. Washington Luiz, km 235, 13565-905 \\ São Carlos - SP, Brasil
}

Recebido em 24/2/10; aceito em 21/6/10; publicado na web em 17/9/10

\begin{abstract}
CO PREFERENTIAL OXIDATION ON CuO/CeO $-\mathrm{TiO}_{2}$ SYSTEM. Cu catalysts supported on $\mathrm{CeO}_{2}, \mathrm{TiO}_{2}$ and $\mathrm{CeO}_{2} / \mathrm{TiO}_{2}$ were prepared by precipitation method and used for preferential oxidation of carbon monoxide contained in a hydrogen flow generated by methane steam reforming. The samples were characterized by XRD, BET and TPR techniques. The catalytic properties were studied in the $50-330^{\circ} \mathrm{C}$ range by using a quartz micro-reactor vertically positioned on an electrical furnace. The results showed that the small copper particles generated with the lower metal content are the most easily reducible and give the best catalytic performance. In respect of support effect, the strong metal-support interaction and the redox characteristics of the $\mathrm{CuO}_{\mathrm{x}}-\mathrm{CeO}_{2}$ series resulted in the best catalytic results, especially with the sample with $1 \%$ copper content.
\end{abstract}

Keywords: PROX-CO; copper; ceria.

\section{INTRODUÇÃO}

Estudos recentes relacionados com a tecnologia de células a combustível, especialmente aqueles envolvendo membranas poliméricas de troca iônica (PEMFC), têm estimulado o interesse pela reação de oxidação preferencial do monóxido de carbono (PROX-CO). Uma vez que as células PEMFC utilizam como fonte de energia o hidrogênio com elevada pureza, a reação de PROX-CO apresenta-se como um meio de purificar o hidrogênio produzido pelas reações de reforma do metano. ${ }^{1-10}$

O método convencional de produção de hidrogênio combina as reações de reforma a vapor do metano com a reação de deslocamento gás-água (WGS - water gas shift reaction), e os gases gerados apresentam a seguinte composição aproximada: $45 \% \mathrm{H}_{2}, 25 \% \mathrm{CO}_{2}$, $10 \% \mathrm{H}_{2} \mathrm{O}, 20 \% \mathrm{~N}_{2}$ e $0,5-1 \%$ CO. ${ }^{1,2}$ Essa quantidade de CO presente no gás de reforma precisa ser removida devido a efeitos adversos que provoca no anodo das células PEMFC, ocasionando a desativação do eletrocatalisador de Pt que está depositado sobre uma camada difusora à base de carbono e teflon (PTFE). Assim, é necessária a remoção do $\mathrm{CO}$, reduzindo seus níveis para valores menores que 10 ppm. ${ }^{1}$ Para alcançar esse nível de concentração, alguns caminhos são estudados: metanação catalítica, separação por membrana de Pd e oxidação preferencial do CO (PROX-CO). ${ }^{1,11}$ Destas, a PROX é a que oferece menor custo na redução dos níveis de $\mathrm{CO}$ sem o consumo excessivo de hidrogênio. ${ }^{1}$

Juntamente com a PROX-CO (Equação 1), a reação indesejada de oxidação do hidrogênio (Equação 2) pode ocorrer, diminuindo a eficiência do processo. ${ }^{1}$

$$
\begin{aligned}
& \mathrm{CO}+1 / 2 \mathrm{O}_{2} \rightarrow \mathrm{CO}_{2} \Delta \mathrm{H}_{298 \mathrm{~K}}^{0}=-283 \mathrm{KJ} / \mathrm{mol} \\
& \mathrm{H}_{2}+1 / 2 \mathrm{O}_{2} \rightarrow \mathrm{H}_{2} \mathrm{O} \Delta \mathrm{H}_{298 \mathrm{~K}}^{0}=-242 \mathrm{KJ} / \mathrm{mol}
\end{aligned}
$$

$\mathrm{O}$ catalisador convencional para a PROX-CO é $\mathrm{Pt} / \gamma-\mathrm{Al}_{2} \mathrm{O}_{3}$, porém diferentes tipos de catalisadores mostraram ser eficientes para a reação. Estes podem envolver fases ativas de metais nobres suportados, como platina e ouro, ou ainda metais não-nobres, como o cobre suportado em óxidos. ${ }^{1,2}$

\footnotetext{
*e-mail: crisqui@gmail.com
}

Os catalisadores de metais nobres exibem boa atividade catalítica em temperaturas entre $150-250{ }^{\circ} \mathrm{C}$ mas, além de apresentarem um alto custo, no geral, são pouco seletivos. ${ }^{3}$ Por outro lado, alguns catalisadores de metais não nobres suportados em óxidos têm se mostrado eficientes aos requisitos da PROX-CO, exibindo também boa atividade e seletividade. ${ }^{3}$

O estudo de catalisadores de cobre suportados em óxidos para a PROX-CO tem trazido resultados interessantes e importantes mostrando, muitas vezes, maior eficiência que os catalisadores de metais nobres. Os que apresentam melhores resultados são os suportados em céria. Nos últimos anos, a céria tem sido muito estudada como suporte em catálise, além de ser um poderoso promotor que modifica propriedades estruturais e eletrônicas de muitos catalisadores usados em importantes reações industriais, tais como, a quebra de óleos pesados em zeólitas, a conversão automotiva, a reforma a vapor e seca do metano e a reação de deslocamento gás-água. ${ }^{3-10,12} \mathrm{O}$ uso da céria como promotor está normalmente relacionado à sua capacidade de armazenar oxigênio e a aspectos estruturais, como a propriedade de aumentar a dispersão dos metais. Na presença de um metal de transição, o tamanho pequeno do cristal de céria favorece a formação de espécies de oxigênio altamente reativas. Estudos recentes mostram que a importância da céria para reação de PROX-CO deve-se ao par redox $\mathrm{Ce}^{+4} / \mathrm{Ce}^{+3}$, que permite ao material agir armazenando oxigênio ou fornecendo-o para reações que estejam ocorrendo em sua superfície. ${ }^{13,14}$

Martinez-Arias et al. estudaram o efeito do suporte sobre a atividade catalítica na reação de PROX-CO de sistemas $\mathrm{CuO} / \mathrm{CeO}_{2}$ e $\mathrm{CuO} /(\mathrm{Ce}, \mathrm{M}) \mathrm{O}_{\mathrm{x}}$, onde $\mathrm{M}=\mathrm{Zr}$ ou Tb. $\mathrm{O}$ catalisador suportado em céria apresentou a melhor atividade e seletividade. Os autores verificaram que os efeitos na conversão são correlacionados com as atividades redox das correspondentes interfaces cobre-óxidos. ${ }^{15}$

Outras evidências sobre a influência do suporte na atividade catalítica foram apresentadas por Ratnasamy et al., que prepararam catalisadores de $\mathrm{CuO}$ suportados em céria, zircônia e céria/zircônia e os testaram na reação de oxidação preferencial do monóxido de carbono. $\mathrm{O}$ composto $\mathrm{CuO}-\mathrm{CeO}_{2}$ exibiu significativo desempenho na oxidação total do $\mathrm{CO}$, apresentando atividade catalítica superior à dos catalisadores $\mathrm{CuO}-\mathrm{ZrO}_{2}$ e $\mathrm{CuO}-\mathrm{CeO}_{2}-\mathrm{ZrO}_{2}$. Isto foi atribuído ao efeito promovido pela céria no sistema $\mathrm{CuO}-\mathrm{CeO}_{2}-\mathrm{ZrO}_{2}$, diminuindo os potenciais redox dos pares $\mathrm{Cu}(\mathrm{II}) / \mathrm{Cu}(\mathrm{I})$ e $\mathrm{Cu}(\mathrm{I}) / \mathrm{Cu}(0)$ na matriz da céria. ${ }^{16}$ 
Desta forma, o objetivo do presente trabalho foi preparar e caracterizar o sistema $\mathrm{CuO} / \mathrm{CeO}_{2}-\mathrm{TiO}_{2}$ e testá-lo na reação de oxidação preferencial do CO. O dióxido de titânio foi utilizado como suporte sozinho ou combinado com a céria. Óxidos inorgânicos, como a titânia, são comumente usados como suportes/promotores de catalisadores, provocando aumento da área superficial e maiores estabilidade térmica e resistência mecânica. ${ }^{17}$

\section{PARTE EXPERIMENTAL}

\section{Preparação dos catalisadores}

A céria foi preparada por precipitação, partindo-se de $\mathrm{CeO}_{2}\left(\mathrm{NO}_{3}\right)_{2} \cdot 6 \mathrm{H}_{2} \mathrm{O}$ e hidróxido de amônio. A temperatura reacional foi ajustada em $70^{\circ} \mathrm{C}$, o sistema foi mantido sob agitação e o $\mathrm{pH}$ controlado em 9. Durante $4 \mathrm{~h}$, a mistura foi deixada para envelhecimento. $\mathrm{O}$ material foi filtrado e lavado, em seguida foi seco durante $12 \mathrm{~h}$ a $60^{\circ} \mathrm{C}$. O sólido obtido foi calcinado a $500^{\circ} \mathrm{C}$, por $4 \mathrm{~h}$ com ar sintético, com fluxo de $80 \mathrm{~mL} \mathrm{~min}^{-1}$. A titânia utilizada foi a P-25 da Degussa. O suporte céria/titânia (1/5) foi preparado por impregnação, com excesso de solvente, calcinado a $500^{\circ} \mathrm{C}$ por $4 \mathrm{~h}$ com fluxo de ar sintético numa vazão de aproximadamente $80 \mathrm{~cm}^{3} \mathrm{~min}^{-1}$, com rampa de aquecimento de $10{ }^{\circ} \mathrm{C} \mathrm{min}^{-1}$.

A adição de cobre foi realizada por impregnação. Os teores mássicos de cobre variaram entre 1,2,5 e 5\% do metal. A fonte de cobre utilizada foi o nitrato de cobre tri-hidratado. Os sólidos foram calcinados a $500{ }^{\circ} \mathrm{C}$ por um período de $4 \mathrm{~h}$, sob fluxo de ar sintético de $80 \mathrm{~mL} \mathrm{~min}^{-1}$ e rampa de aquecimento de $10^{\circ} \mathrm{C} \mathrm{min}^{-1}$.

\section{Caracterização dos catalisadores}

Os ensaios de DRX dos sólidos foram realizados pelo método do pó em equipamento Rigaku Multiflex - 2KW, com radiação $\mathrm{CuK} \alpha$, velocidade de varredura de $2^{\circ} \min ^{-1}$ e faixa de varredura de $10-80^{\circ}$.

A área superficial específica dos catalisadores foi medida pela adsorção física de nitrogênio sobre o catalisador, pelo método BET (modelo proposto por Brunaeur, Emmett e Teller). ${ }^{18}$ Os dados experimentais foram obtidos em um equipamento Quantachrome Nova modelo 1200 .

As análises de redução à temperatura programada foram realizadas em um equipamento Micromeritics Pulse Chemisorb 2705 equipado com detector de condutividade térmica, vazão volumétrica da mistura $5 \% \mathrm{H}_{2} / \mathrm{N}_{2}$ de $30 \mathrm{~mL} \mathrm{~min}^{-1}$ e temperatura de análise variando da ambiente até $800^{\circ} \mathrm{C}$, com rampa de aquecimento de $10^{\circ} \mathrm{C} \mathrm{min}^{-1}$.

\section{Ensaios catalíticos}

A atividade catalítica foi testada usando-se um reator de leito fixo, sob pressão atmosférica.

Os sólidos foram ativados in situ, com fluxo de $30 \mathrm{~mL} \mathrm{~min}{ }^{-1}$ de hidrogênio a $300^{\circ} \mathrm{C}$, durante $2 \mathrm{~h}$. A temperatura reacional variou de 50 a $300^{\circ} \mathrm{C}$

Os ensaios catalíticos foram realizados com alimentação de monóxido de carbono (4\%), ar sintético (2\%), hidrogênio (em excesso) e nitrogênio.

Os efluentes foram analisados em cromatógrafo para análise de gases Varian 3800, equipado com dois detectores de condutividade térmica.

As quantidades de $\mathrm{CO}$ e de $\mathrm{O}_{2}$ convertidas em produtos foram calculadas pela Equação 3:

$$
X_{i}(\%)=\frac{\left(n_{i} i-n_{f} i\right)}{n_{i} i} \times 100
$$

onde $\mathrm{X}_{\mathrm{i}}$ é a conversão do componente $\mathrm{i}, \mathrm{n}_{\mathrm{i}} \mathrm{i}$ o número de mols inicial do componente i e $\mathrm{n}_{\mathrm{f}} \mathrm{i}$ é o número de mols final do componente $\mathrm{i}$.

A seletividade para formação de $\mathrm{CO}_{2}$ foi calculada pela Equação 4:

$$
S_{\mathrm{O}_{2}, \mathrm{CO}_{2}}(\%)=\frac{\left(n_{i} \mathrm{CO}-n_{f} \mathrm{CO}\right)}{0,5 x\left(n_{i} \mathrm{O}_{2}-n_{f} \mathrm{O}_{2}\right)} \times 100
$$

onde $\mathrm{S}_{\mathrm{O}_{2} \mathrm{CO}_{2}}(\%)$ é a seletividade do oxigênio para a oxidação do $\mathrm{CO}$ a $\mathrm{CO}_{2} ; \mathrm{n}_{\mathrm{i}} \mathrm{CO}$ o número de mols inicial de $\mathrm{CO} ; \mathrm{n}_{\mathrm{f}} \mathrm{CO}$ o número de mols final de $\mathrm{CO} ; \mathrm{n}_{\mathrm{i}} \mathrm{O}_{2}$ o número de mols inicial de oxigênio e $\mathrm{n}_{\mathrm{f}} \mathrm{O}_{2}$ o número de mols final de oxigênio.

\section{RESULTADOS E DISCUSSÃO}

\section{Caracterização dos catalisadores}

\section{Difração de Raios-X}

A Figura 1 mostra os resultados de DRX dos suportes, enquanto a Figura 2 apresenta os resultados referentes aos catalisadores suportados. Picos relativos à fase cerianita (111) com estrutura cúbica de face centrada foram observados no suporte céria e céria/titânia. Observam-se ainda picos referentes às fases anatase (101) e rutila (110) do suporte $\mathrm{TiO}_{2}-\mathrm{P} 25$.

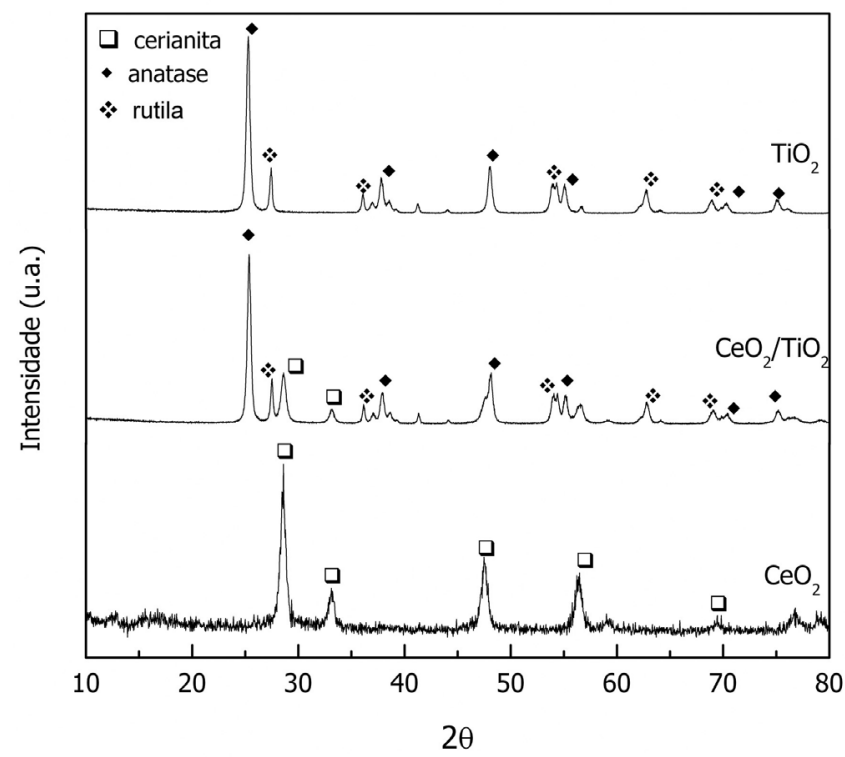

Figura 1. Difratograma dos suportes

Pode-se verificar, na Figura 2, que os catalisadores suportados apresentaram basicamente picos de difração referentes aos suportes utilizados. A presença da fase $\mathrm{CuO}$ não foi observada nos catalisadores com teor metálico de $1 \%$, sugerindo que as espécies do óxido de cobre estão altamente dispersas no suporte, resultando em pequeno tamanho de cristal, que não pode ser detectado pela técnica. ${ }^{11,15,19}$

Através dos picos de maior intensidade de cada amostra, foi possível calcular o tamanho dos cristalitos, usando a Equação de Scherrer. Os resultados obtidos estão apresentados na Tabela 1.

Observa-se, nestes resultados, que os sólidos suportados em titânia possuem tamanhos de cristalitos maiores. Este fato é devido ao tamanho do cristalito da titânia ser superior aos da céria e céria/ titânia. Como esperado, o suporte céria/titânia e os catalisadores nele suportados apresentaram cristalitos de tamanho intermediário aos da titânia e céria. 


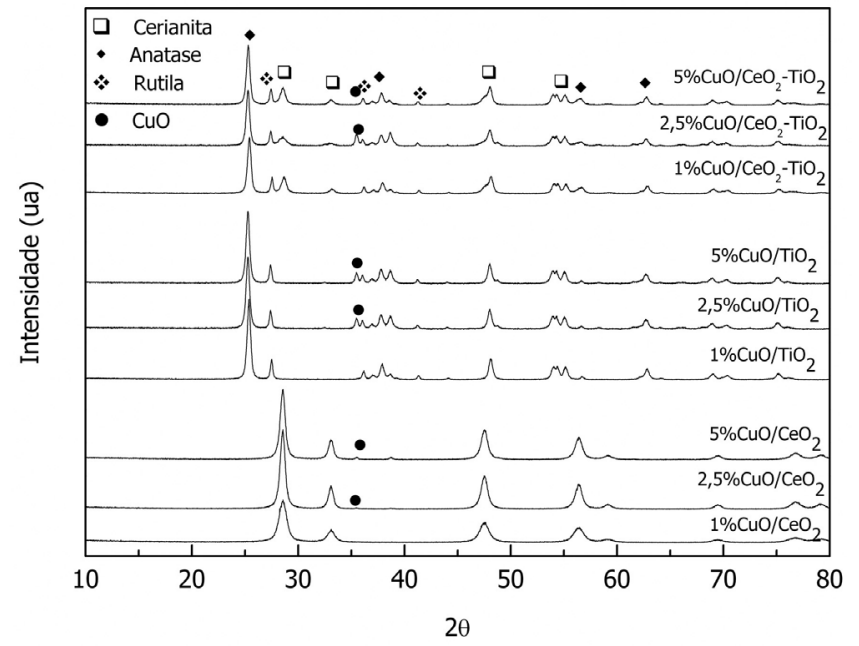

Figura 2. Difratograma dos sólidos suportados

Tabela 1. Tamanho de cristalito dos sólidos calcinados

\begin{tabular}{lcc}
\hline Sólido & Fase & $\begin{array}{c}\text { Tamanho do } \\
\text { Cristalito (nm) }\end{array}$ \\
\hline $\mathrm{TiO}_{2}-\mathrm{P} 25$ & Anatase (101) & 33 \\
$\mathrm{CeO}_{2}$ & Cerianita (111) & 11 \\
$\mathrm{CeO}_{2} / \mathrm{TiO}_{2}$ & Rutila(110) & 23 \\
$\mathrm{CuO} / \mathrm{CeO}_{2}-1 \%$ & Cerianita (111) & 15 \\
$\mathrm{CuO} / \mathrm{CeO}_{2}-2,5 \%$ & Cerianita (111) & 10 \\
$\mathrm{CuO} / \mathrm{CeO}_{2}-5 \%$ & Cerianita (111) & 15 \\
$\mathrm{CuO} / \mathrm{TiO}_{2}-1 \%$ & Anatase (101) & 25 \\
$\mathrm{CuO} / \mathrm{TiO}_{2}-2,5 \%$ & Anatase (101) & 23 \\
$\mathrm{CuO} / \mathrm{TiO}_{2}-5 \%$ & Rutila (110) & 22 \\
$\mathrm{CuO} / \mathrm{CeO}_{2}-\mathrm{TiO}_{2}-1 \%$ & Anatase (101) & 24 \\
$\mathrm{CuO} / \mathrm{CeO}_{2}-\mathrm{TiO}_{2}-2,5 \%$ & Anatase (101) \\
$\mathrm{CuO} / \mathrm{CeO}_{2}-\mathrm{TiO}_{2}-5 \%$ & Anatase (101) & 22 \\
\hline
\end{tabular}

\section{Área superficial específica (B.E.T)}

A Tabela 2 apresenta os resultados das análises texturais dos suportes e dos catalisadores suportados. Tanto os catalisadores quanto os suportes calcinados apresentam baixa área superficial específica. Nos catalisadores suportados em titânia e céria/titânia, a adição do metal não provocou mudanças na área superficial, quando comparada à titânia e à céria/titânia sozinhas. Por outro lado, a adição do metal ao suporte céria provocou decréscimo no valor da área superficial, exceto para o catalisador $\mathrm{CuO} / \mathrm{CeO}_{2}(1 \%)$, onde essa diminuição não foi observada. $\mathrm{O}$ decréscimo de área com adição da fase ativa pode estar relacionado com bloqueio de poros de menor tamanho do suporte.

\section{Redução a temperatura programada.}

Os perfis de RTP dos sólidos são apresentados na Figuras 3, 4 e 5. Na Figura 3, observa-se que no suporte $\mathrm{CeO}_{2}$ a redução começou em $495^{\circ} \mathrm{C}$, onde aparece um primeiro ombro. Dois picos mais característicos aparecem em $631^{\circ} \mathrm{C}$ e $878^{\circ} \mathrm{C}$. O pico a $631^{\circ} \mathrm{C}$ é identificado como sendo devido à redução da céria superficial, enquanto o pico a $878{ }^{\circ} \mathrm{C}$, de maior intensidade, pode ser descrito como a redução do $\mathrm{CeO}_{2}$ mássico. ${ }^{16,20}$

De acordo com a literatura, a redução do cobre presente em óxidos suportados em céria é caracterizada pela presença de dois picos característicos, que são atribuídos a espécies de cobre finamente dispersas no suporte e espécies de cobre do $\mathrm{CuO}$ bulk (baixa e alta temperatura,
Tabela 2 . Resultados das análises texturais dos sólidos

\begin{tabular}{lcc}
\hline Sólido & $\begin{array}{c}\text { Teor de metal teórico } \\
(\%)\end{array}$ & $\begin{array}{c}\text { Área superficial específica } \\
\left(\mathrm{m}^{2} / \mathrm{g}\right)\end{array}$ \\
\hline $\mathrm{CeO}_{2}$ & --- & 63 \\
$\mathrm{TiO}_{2}$ & --- & 69 \\
$\mathrm{CeO}_{2} / \mathrm{TiO}_{2}$ & --- & 47 \\
$\mathrm{CuO} / \mathrm{CeO}_{2}$ & 1 & 60 \\
& 2,5 & 27 \\
& 5 & 26 \\
$\mathrm{CuO} / \mathrm{TiO}_{2}$ & 1 & 43 \\
& 2,5 & 45 \\
& 5 & 44 \\
$\mathrm{CuO} / \mathrm{CeO}_{2}-\mathrm{TiO}_{2}$ & 1 & 50 \\
& 2,5 & 44 \\
& 5 & 46 \\
\hline
\end{tabular}

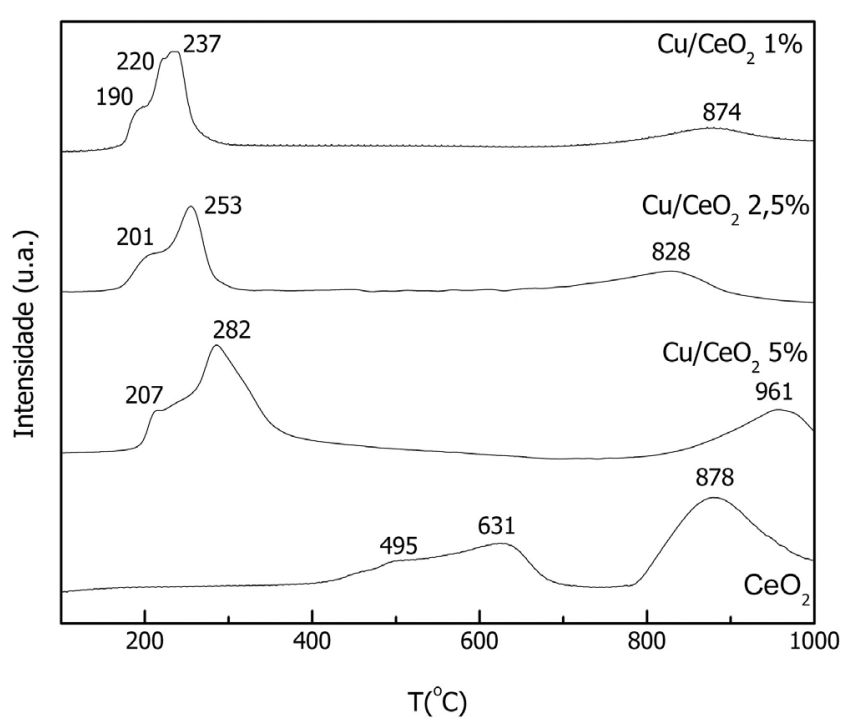

Figura 3. RTP da céria e dos sólidos suportados em céria

respectivamente). A redução depende do tamanho de partículas do sólido; assim, $\mathrm{CuO}$ com partículas grandes se reduze em temperaturas maiores, pois requer maior quantidade de energia, enquanto pequenas partículas de $\mathrm{CuO}$ se reduzem a temperaturas mais baixas. ${ }^{19-23}$ Já a céria apresenta picos de redução que são relacionados com redução do óxido de cério na superfície ou com o bulk de oxigênio (também chamada de céria bulk). ${ }^{24}$ Polster et al. relatam ainda que existem diferentes espécies de oxigênio da céria e do óxido de cobre que se reduzem a diferentes temperaturas no sólido $\mathrm{CuO}_{\mathrm{x}}-\mathrm{CeO}_{2}$, mas estas são as mesmas espécies de oxigênio em cada óxido puro. ${ }^{25}$

O perfil de RTP da titânia, mostrado na Figura 4, apresenta pequenas ondulações nas temperaturas de 500 e $600{ }^{\circ} \mathrm{C}$, não chegando a caracterizar um pico. Isto indica que, em toda a faixa experimental observada, a titânia não foi reduzida, resultados estes que coincidem com os relatados por Zhu et al. ${ }^{20}$

O perfil de RTP da céria/titânia, exibido na Figura 5, mostra um pico de redução a $858{ }^{\circ} \mathrm{C}$ e três pequenos ombros a 368,518 e $602{ }^{\circ} \mathrm{C}$. Comparando este perfil com os da céria e da titânia isoladas, pode-se afirmar que todos os picos são referentes à presença da céria; no entanto, os picos que aparecem no suporte céria/titânia estão deslocados para temperaturas menores quando comparados com os da céria e essa facilidade relativa está relacionada com o tamanho dos cristalitos presentes nos dois suportes, facilitando ou dificultando a redução..$^{20,23}$ 


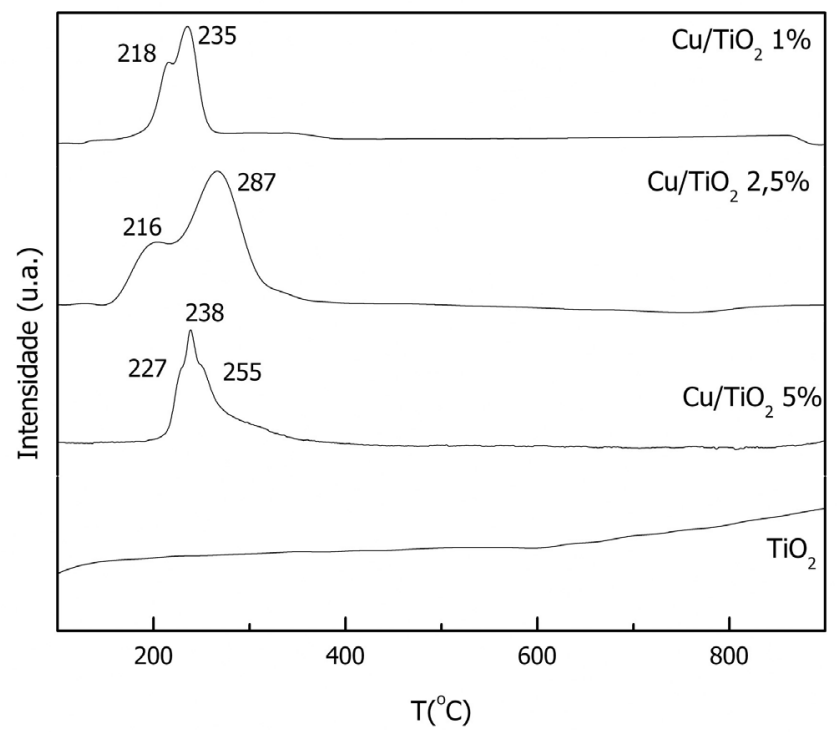

Figura 4. RTP do suporte titânia e dos sólidos suportados em titânia

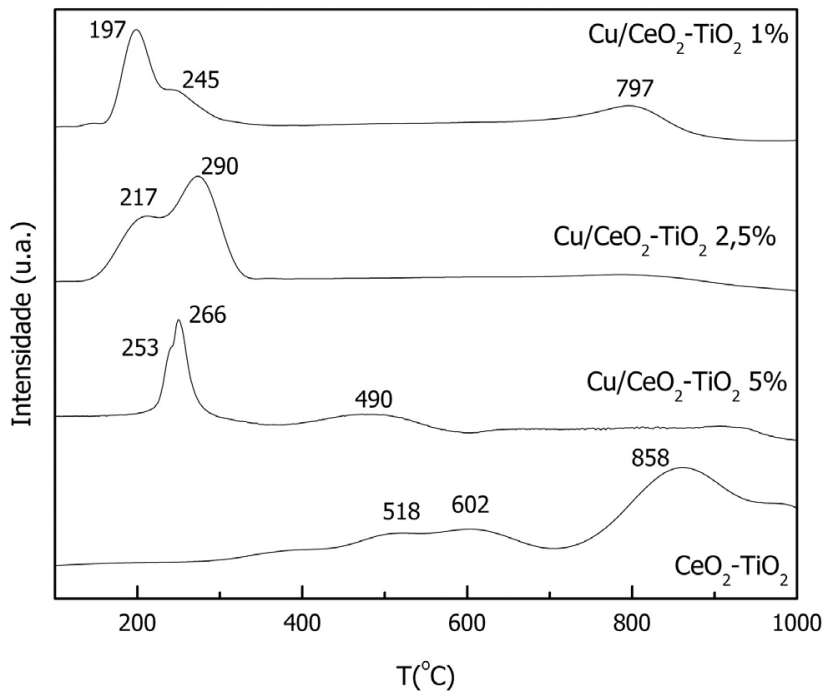

Figura 5. RTP do suporte céria/titânia e dos sólidos suportados em cérial titânia

Nos catalisadores suportados, notam-se dois picos em temperaturas baixas, que se referem à redução do cobre. Apenas os catalisadores $\mathrm{CuO} / \mathrm{CeO}_{2}(5 \%)$ e $\mathrm{CuO} / \mathrm{TiO}_{2}(5 \%)$ mostram três picos de redução referentes ao metal, sendo que o pico a menor temperatura corresponde à redução das espécies de $\mathrm{CuO}$ com partículas pequenas ligadas à superfície do suporte e os picos em temperaturas maiores são devidos à redução do $\mathrm{CuO}$ bulk, presente em partículas grandes ligadas ao suporte. ${ }^{16,26-28}$

Nos catalisadores suportados em céria e céria/titânia, além dos picos de redução referentes ao metal, outro pico, a temperaturas maiores, pode ser observado e deve-se à redução do óxido de cério mássico.

Os catalisadores suportados em titânia só apresentam picos de redução do óxido de cobre, uma vez que o suporte é estável até a temperatura de $1000{ }^{\circ} \mathrm{C} . .^{27-29}$

Relacionando as temperaturas de redução referentes ao $\mathrm{CuO}$ com o teor mássico do metal em cada amostra, verifica-se que o aumento do teor de cobre provoca um deslocamento dos picos, embora pequeno, para menores temperaturas. Isso acontece com todos os catalisadores suportados.

\section{Atividade catalítica}

As Figuras 6 e 7 apresentam os resultados de atividade catalítica e seletividade relativos aos catalisadores testados na reação de PROX-CO.

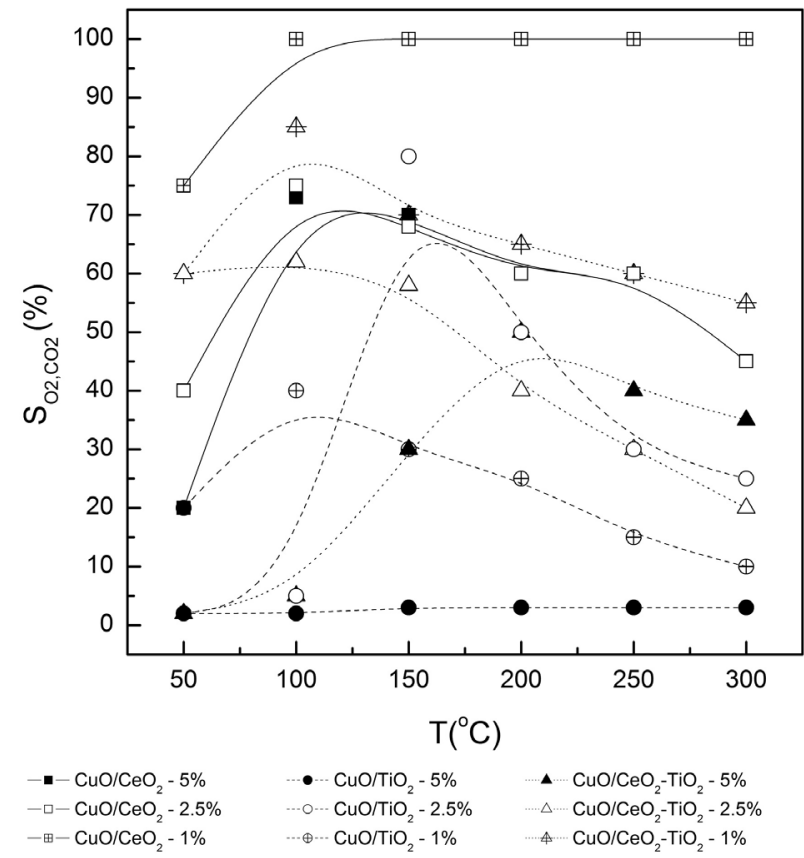

Figura 6. Atividade catalítica dos catalisadores suportados

Observa-se, na Figura 6, que os sólidos suportados em céria foram mais ativos que os demais, em toda a faixa de temperaturas. Para estes, a diminuição do teor metálico favoreceu a conversão do $\mathrm{CO}$ a $\mathrm{CO}_{2}$, observando-se que à temperatura de $50{ }^{\circ} \mathrm{C}$, o catalisador $\mathrm{CuO} / \mathrm{CeO}_{2}$ $-1 \%$ apresentou conversão de aproximadamente $100 \%$. Os sólidos $\mathrm{CuO} / \mathrm{CeO}_{2} 2,5$ e $5 \%$ apresentam atividade catalíticas semelhantes, com um máximo de conversão próximo a $100{ }^{\circ} \mathrm{C}$ e com acentuada queda à medida que a temperatura de reação diminui.

Nos sólidos suportados em titânia o comportamento é análogo, no entanto, as taxas de conversão são menores. Observa-se, com o catalisador com teor metálico de $1 \%$, um máximo de conversão a $100{ }^{\circ} \mathrm{C}$ em aproximadamente $60 \%$ e com o catalisador $\mathrm{CuO} / \mathrm{TiO}_{2}$ $2,5 \%$ o máximo aparece a $150{ }^{\circ} \mathrm{C}$. Em temperaturas inferiores a estes máximos, há acentuada queda da atividade catalítica.

Com os catalisadores suportados em céria/titânia nota-se também que a diminuição do teor metálico leva a maiores taxas de conversão. O sólido com menor teor metálico foi o mais ativo para a PROX-CO em todo o intervalo de temperaturas testado. Nota-se um máximo a $100{ }^{\circ} \mathrm{C}$ e, com a diminuição da temperatura, um acentuado decréscimo. Ponto de máximo de conversão também é observado para os outros catalisadores suportados em céria/titânia.

A conversão do $\mathrm{O}_{2}$ foi constante e igual a $100 \%$, para todos os catalisadores, em todo o intervalo experimental (resultados não apresentados).

A Figura 7 apresenta os resultados de seletividade obtidos com os catalisadores suportados. Além de ser o mais ativo para a PROX-CO, o composto $\mathrm{CuO} / \mathrm{CeO}_{2} 1 \%$ foi também o mais seletivo apresentando, acima de $100{ }^{\circ} \mathrm{C}$, seletividade de $100 \%$. As curvas de seletividade com $\mathrm{CuO} / \mathrm{CeO}_{2} 2,5$ e $5 \%$ foram semelhantes, com máximos a $100^{\circ} \mathrm{C}$ e $70 \%$ de conversão. Os catalisadores suportados em céria/titânia apresentaram esta mesma tendência. Por outro lado, a seletividade do composto $\mathrm{CuO} / \mathrm{TiO}_{2} 2,5 \%$ foi superior à dos outros catalisadores, com seletividade máxima de $80 \%$ a $150{ }^{\circ} \mathrm{C}$. O catalisador $\mathrm{CuO} / \mathrm{TiO}_{2}$ 


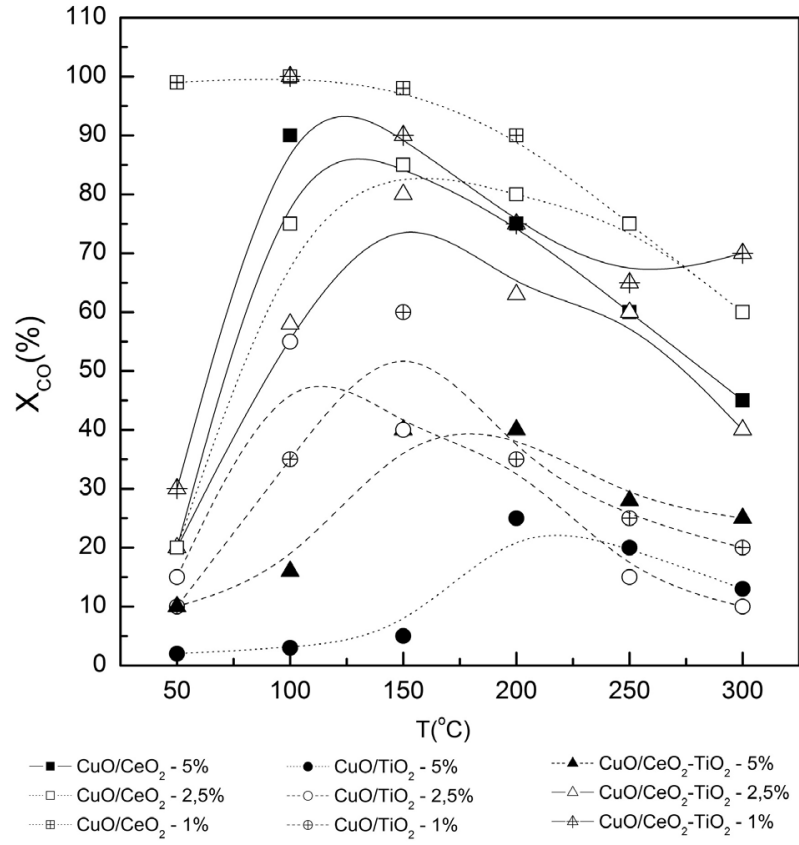

Figura 7. Seletividade dos catalisadores suportados

$5 \%$ apresentou seletividade baixa e praticamente constante em toda a faixa de temperaturas de reação.

O que pode ser notado é que a conversão sobre catalisadores suportados em céria é superior àquela dos suportados em titânia ou céria/titânia. Este fato pode ser atribuído ao efeito promotor da céria, relacionado à sua capacidade de mudança do estado de oxidação. Neste processo, o oxigênio se insere nas vacâncias da céria parcialmente reduzida, mudando seu estado de oxidação. Em seguida, $\mathrm{CO}$ adsorvido no cobre reage com um oxigênio doado pela céria, gerando novamente uma vacância. Este processo se repete continuamente. ${ }^{8,30-32}$ Além disso, Manzoli et al. observaram que a boa atividade destes catalisadores está relacionada com as espécies de cobre superficiais e induzidas pela interação destas com a céria (forte interação metalsuporte), fator determinante para a boa atividade destes sólidos. ${ }^{32}$

Os sólidos suportados em céria/titânia tiveram atividade catalítica satisfatória, no entanto, inferiores aos sólidos suportados em céria.

Outro fato importante a ser observado é que os catalisadores com teor metálico de $1 \%$ apresentaram valores mais altos de conversão e de seletividade. Como discutido anteriormente, o pequeno tamanho de partículas metálicas apresentado por estes catalisadores pode ser considerado como a razão desse comportamento. A amostra com 1\% de cobre também foi a que apresentou maior área superficial específica, sendo este outro fator que contribui para uma maior dispersão dos sítios catalíticos.

\section{CONCLUSÕES}

A forte interação metal-suporte existente nos sólidos $\mathrm{CuO}_{\mathrm{x}}-\mathrm{CeO}_{2}$, aliada às características redox do par cobre/céria, podem ser apontadas como responsáveis por um melhor desempenho apresentado por estes sólidos quando comparados a catalisadores $\mathrm{CuO}_{x} / \mathrm{TiO}_{2}$ e $\mathrm{CuO}_{\mathrm{x}}-\mathrm{CeO}_{2} /$ $\mathrm{TiO}_{2}$ preparados por impregnação. Os perfis de RTP revelam ainda que as espécies de cobre são mais facilmente reduzidas quanto menor o teor deste metal adicionado ao suporte. Este fato está relacionado ao menor tamanho de partícula apresentado pelos sólidos com teores metálicos mais baixos e responde pelo melhor desempenho catalítico dos mesmos, especialmente daqueles suportados em céria. Os resultados dos ensaios de DRX e BET dão suporte a esta constatação, mostrando que o aumento do tamanho de partícula é diretamente proporcional ao teor metálico.

\section{AGRADECIMENTOS}

Ao CNPq e à Capes pelos auxílios e bolsa concedidos.

\section{REFERÊNCIAS}

1. Chin, S. Y.; Alexeev, O. S.; Amiridis, M. D.; Appl. Catal., A 2005, 286, 157.

2. Ghenciu, A. F.; Current Opinion in Solid State and Materials Science 2000, 6, 389.

3. Schonbrod, B.; Marino, F.; Baronetti, G.; Laborde, M.; Int. J. Hydrogen Energy 2009, 34, 4021.

4. Gurbania, A.; Ayastuya, J. L.; Gonzalez-Marcosa, M. P.; Herrerob, J. E.; Guilb, J. M.; Gutierrez-Ortiza, M. A.; Int. J. Hydrogen Energy 2009, 34, 547.

5. Ayastuy, J. L.; Gurbani, A.; Gonzalez-Marcos, M. P.; Gutierrez-Ortiz, M. A.; Int. J. Hydrogen Energy 2010, 35, 1232.

6. Gomez-Cortes, A.; Marquez, Y.; Arenas-Alatorre, J.; Diaz, G.; Catal. Today 2008, 133, 743.

7. Yi, G.; Xu, Z.; Guo, G.; Tanaka, K.; Yuan, Y.; Chem. Phys. Lett. 2009, 479, 128.

8. Moretti, E.; Storaro, L.; Talon, A.; Lenarda, M.; Catal. Commun. 2009, 10,522 .

9. Liu, Z.; Zhou, R.; Zheng, X,; J. Nat. Gas Chem. 2008, 17, 125.

10. Martinez-Arias, A.; Gamarra, D.; Fernadez-Garcia, M.; Hornes, A.; Bera, P.; Koppany, Z.; Schay, Z.; Catal. Today 2009, 143, 211.

11. Liu, Y.; Fu, Q.; Stephanopoulos, M. F.; Catal. Today 2004, 93, 241.

12. Muccillo, E. N. S.; Porfirio, T. C.; Tadokoro, S. K.; Rey, J. F. Q.; Rocha, R. A.; Stei, M. C.; Muccillo, R.; Revista Cerâmica 2005, 51, 157.

13. Cataluna, R.; Baibich, I.; M.; Dallago, R. M.; Quim. Nova 2001, 24, 55.

14. Martınez-Arias, A.; Fernandez-Garcıa, M.; Soria, J.; Conesa, J. C.; J. Catal. 1999, 182, 367.

15. Martinez-Arias, A.; Hungria, A. B.; Fernandez-Garcia, M.; Conesa, J. C.; Munuera, G.; J. Power Sources 2005, 151, 32.

16. Ratnasamy, R.; Srivinas, D.; Satyanarayana, C. V. V.; Manikandan, P.; Senthil Kumaran, R. S.; Sachin, M.; Shetti, V. N.; J. Catal. 2004, 221, 455.

17. Bouzoubaa, A.; Markovits, A.; Calatayud, M.; Minot, C.; Surf. Sci. 2005, 583, 107.

18. Brunauer, S.; Emmett, P. H.; Teller, E. J.; J. Am. Chem. Soc. 1938, 60, 309.

19. Djinovic, P.; Batista, J.; Pintar, A.; Catal. Today 2009, 147S, S191.

20. Zhu, H.; Qin, Z.; Shan, W.; Shen, W.; Wang, J.; J. Catal. 2004, 225, 267.

21. Giordano, F.; Trovarelli, A.; Leitenburg, C.; Giona, M.; J. Catal. 2000, 193,273

22. Zhang, Y.; Liang, H.; Yu, X.; Liu, Y.; Catal. Commun. 2009, 10, 1432.

23. González, I. D.; Navarro, R. M.; Wen, W.; Marinkovic, N.; Rodriguez, J. A.; Rosa, F.; Fierro, J. L. G.; Catal. Today 2010, 149, 372.

24. Liu, Z.; Zhou, R.; Zheng, X.; J. Mol. Catal. A: Chem. 2007, 267, 137.

25. Polster, C. S.; Nair, H.; Baertsch, C. D.; J. Catal. 2009, 266, 308.

26. Tang, X.; Zhang, B.; Li, Y.; Xu, Y.; Xin, Q.; Shen, W.; Catal. Today 2004, 93, 191.

27. Xiaoyuana, J.; Guanglieb, L.; Renxiana, Z.; Jianxina, M.; Yua, C.; Xiaoming, Z.; Appl. Surf. Sci. 2001, 173, 208.

28. Luo, M.; Zhong, Y.; Yuan, X.; Zheng, X.; Appl. Catal., A 1997, 162, 121.

29. Edmak, G.; Hocevar, S.; Levec, J.; J. Catal. 2004, 222, 87.

30. Zhu, P.; Li, J.; Zuo, S.; Zhou, R.; Surf. Sci. 2008, 2903.

31. Moretti, E.; Storaro, L.; Talon, A.; Lenarda, M.; Catal. Commun. 2009, 522.

32. Manzoli, M.; Monte, R. D.; Boccuzzi, F.; Cluccia, S.; Kaspa, J.; Appl. Catal., B 2005, 61, 192. 